\title{
Optimization of Blood Pressure Waveforms using Adaptive, Bandpass Filters and Wavelet Methods in MATLAB
}

\author{
Shalini S, Mutamilan S, Monisha R, Nithish M
}

\begin{abstract}
Cardiac catheterization is one of the most important way to examine the hemodynamics of a particular patient. In this technique, the whole part of the blood pressure waveforms can be captured and recognized by the cardiologist. These types of measurements are classified by the respiration and fluid filled in catheter, by means of this technique. By this, the measurement will be more accurate, when the artifacts in the waveforms has been removed. In this report we mainly focuses upon the effects of the respiratory artifacts in which the blood pressure signals can be accompanied during the process of catheterization. In this particular project, we use four methods, they are two adaptive filters, one wavelet based method and standard bandpass filters are determined. The blood pressure waveform includes systolic and diastolic pressures. All the above mentioned categories and its methods has been implemented in MATLAB and validated.
\end{abstract}

Keywords : Adaptive filter, bandpass filter, cardiac catheterization, hemodynamics, systolic and diastolic pressures and wavelet methods.

\section{INTRODUCTION}

As much as technology has been improved for health monitoring system, which can be communicated wirelessly. These technologies requires less power consumption. But, the development of these kinds of technologies are essential and they can able to help in abnormalities. By means of monitoring continously, the systems can provide warning signals in emergency situation of the medical fields. Hence, the rate of the pulse and the blood pressure can be continuously monitored and measured continuously. Mostly, pulse rate and the blood pressure is measured using hand cuff devices. The proposed method consists of compensating respiratory artifacts in blood pressure waveforms by means of cardiac catheterization process in hemodynamics. By using the techniques of adaptive and bandpass filters and wavelet transform methods, the blood pressure of the human can able to continuously monitored.

Revised Manuscript Received on March 11, 2020.

* Correspondence Author

Shalini.S ME*, Assitant professor Department of ECE, Sri Krishna College of Technology, Coimbatore.

Mutamilan S, studying, Electronics and communication Engineering, Sri Krishna College of Technology, Coimbatore.

Monisha R, studying Electronics and communication Engineering in Sri Krishna College of Technology, Coimbatore.

Nithish M, studying Electronics and communication Engineering in Sri Krishna College of Technology, Coimbatore. His area of interest is Automation Electronics.

(C) The Authors. Published by Blue Eyes Intelligence Engineering and Sciences Publication (BEIESP). This is an open access article under the CC BY-NC-ND license (http://creativecommons.org/licenses/by-nc-nd/4.0/)
Blood pressure is the continous measurement of all the blood vessels during the blood circulation, which can decreases as it moves from arteries to capillaries inside the heart, and further it can directed towards the heart through veins. Eventually, by means of calculating the rate of heartbeat, the measurement of the blood pressure can be varies from systolic to diastolic pressures. When the blood travels through the arterial circulation by the contraction of the heart at highest rate of pressure is known as the systolic blood pressure, while the blood travels through the arterial circulation at lower rate is known as diastolic blood pressure. In this the pressure is calculated at the lowest rate of arterial circulation pressures. Usually, the blood pressure and the pulse rate can be measured using sphygmomanometer and also manometer is a device, in which the circulating pressures can be determined by the height of a column of mercury. In today's modern technology the blood pressure can be measured via various wireless components. Also, the blood pressure values are determined in terms of millimeters of mercury(mmHg). In this proposed method, the adaptive and bandpass filters are used to remove the artifacts in the blood pressure waveforms,by means of removing these artifacts, the cardiologist can view the accurate form blood pressure waveforms.

\section{LITERATURE SURVEY}

Reza Malekain and Johan Wannenburg has proposed a Body Sensor Network for Mobile Health Monitoring, a diagnosis and Anticipating System in 2015[1]. Here, the blood pressure techniques which is of direct and indirect methods has been discussed. Johnson, A.M Jegan, \& Mary X. A, proposed a performance of the measure of heart rate by means of PPG signal can be calculated [2]. Z. Zhou, L.Xu, K.Chen and X.Zhang has proposed a new method for locating the featured points in pulse wave using wavelet transfor in 2009[3], here the representation of the pulse waveform by means of wavelet method has been determined. M.Q.H Meng Z. Shen, C.Hu has proposed a pulse wave filter method based on adaptive algorithm and wavelet transform softreshhold in 2010[4]. S.S.Ahrabi has commenced the mathematical modeling of flow of blood from an eccentric catheterized artery [5], in this the catheterized artery waveforms can be determined by means of blood flow. S. I. Zhen-Zhen has proposed an application and advancement of and wavelet transform in signal de-noising and fourier transform[6]. L. Kong has proposed a MATLAB based Wavelet transform analysis[7]. 
In this, the wavelet transform functions can be analysed by means of wavelet methods. J.R.C Jansen, S.A Hoeskel, J.A Blom and J.J Screuder has proposed a correction obtained in the pulmonary blood pressure signals of the respiratory artifacts of the particular ventilated patients in 1996 [8]. K.H. Chon, M. Elstad, K.Toska, E.A. Raeder and R.J Cohen. Has propsed a Respiratory sinus arrhythmia which can emphasizes the opposite effects on systolic pressures in 2001 [9]. In this method the determination of arrhythmia has been obtained. S.T. Higano, E. Azrak, N. K. Tahirkheli, and M. J. Kern has proposed a method of influence of respiratory dynamics on ventricular pressures. It's a cardiovascular and catheterization interventions in 1999 [10].In this method we can able to adopt the interventions of catheterization and cardiovascular E.Ruokonen, I.Korhonen, J.Parkka and J.Takala has proposed a method for optimization of pulmonary capillary pressure in 1999. In this method the capillary pressures during the pulmonary process can be obtained.

\section{METHODS AND MATERIALS}

\section{A. Blood pressure waveforms}

Eventually, there are three different blood pressure waveforms has to be measured during a cardiac catheterization those pressures are atrial, ventricular and arterial pressure. Out of these, ventricular and arterial pressures are has to be discussed here. Both of the pressures will be comprised with same shape, but differs with the amplitude . In each period, the waveform of ventricle contracts with the rise of higher pressure, then it is termed as systolic pressure. By contempting the contraction of the system, the pressure gets lower, then it is known as diastolic pressure(dp). In the ventricular blood pressure waveform, two distinct diastolic pressures can be measured. The begin-diastolic pressure is the pressure by which the beginning stage of the diastolic pressure at the particular diastolic period has been measured, while the end-diastolic pressure is the measurement at which the end stage of the diastolic pressures. In this arterial blood pressure waveforms, only one pressure point may be present, which is known as diastolic pressure, thus the lowest pressure can be determined by the time of catheterization.

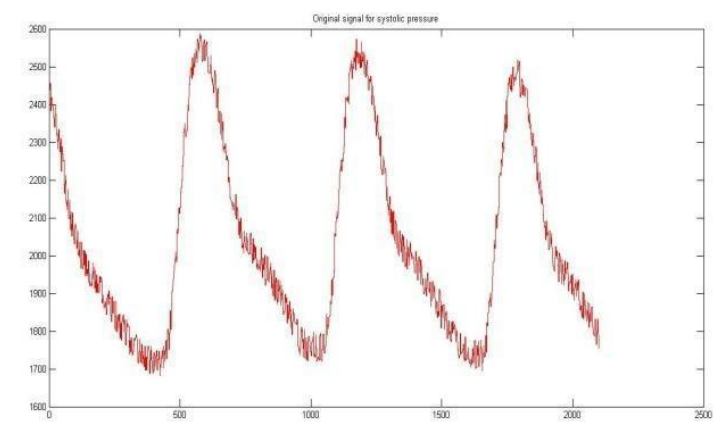

Fig.1.Original signal for systolic pressure

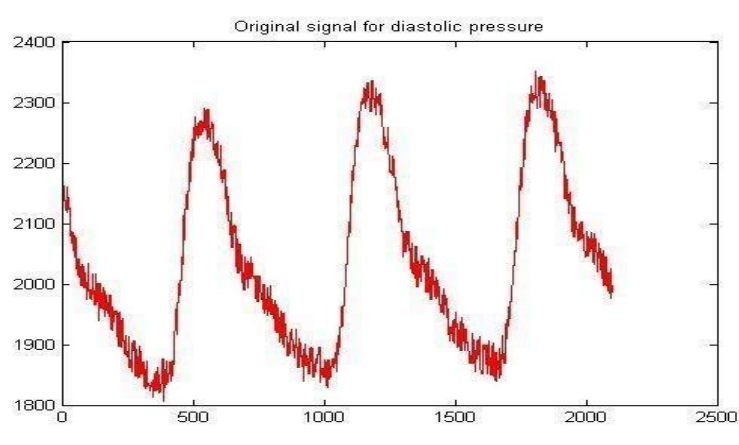

Fig.2.Original signal for diastolic pressure

\section{B. Respiratory signals}

The respiratory signal measurement consists of the level of CO2 and air content in it. This will result the waveform with a clear cut by means of corrulating the entire waveforms. The respiratory signal also classified into two parts,they are respiratory signals at systolic and diastolic end pressures.

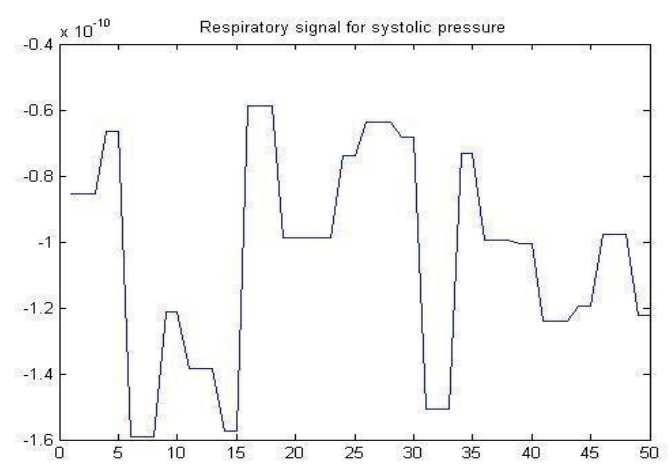

Fig.3.Respiratory signal for systolic pressure

\section{Electrocardiogram(ECG)}

The measurement of ECG determines the continuous heart rate of the patient. It is the reading of electrical heart rate. It is used in instantenous heart rate and the high peak is denoted by $R$, thus the rate of the length in the pressure can be measured via $\mathrm{R}-\mathrm{R}$ variations.

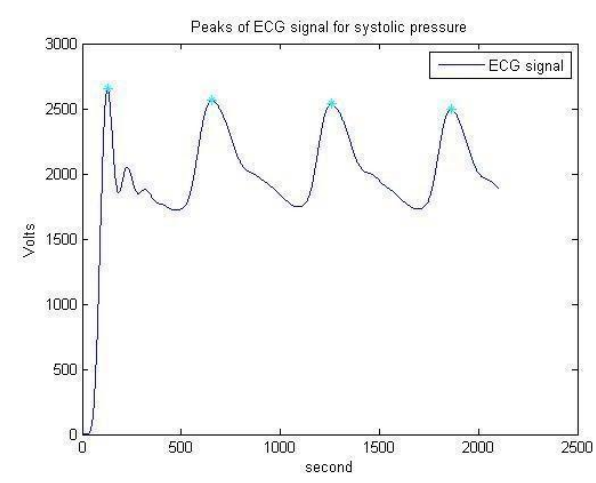

Fig.5.Peaks of ECG for systolic pressure 


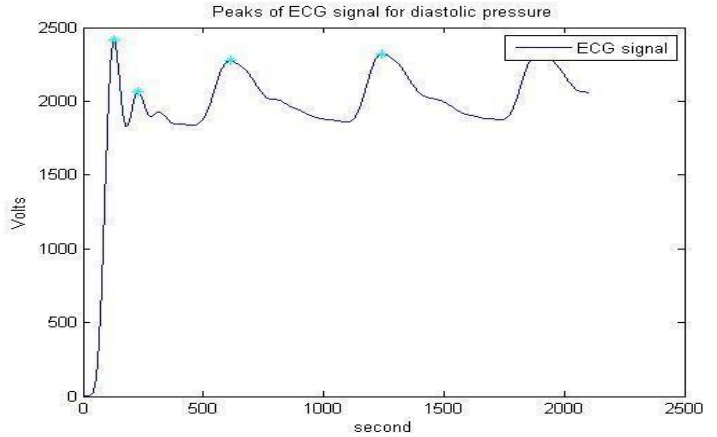

Fig.6.Peaks of ECG signal for diastolic pressure

\section{Respiratory Sinus Arrhythmia(RSA)}

RSA can depends upon the respiratory signals which can affects the rate of blood pressure. RSA is a variation synchronous of heart-rate with respect to the respiration. At corresponding ratings, there will be a decrease or increase in heart-rates. The effects of RSA leads to the fluctuations in the arterial blood pressure waveform, thus the heart-rate can be affected. Also, the RSA is one of the mechanism for keeping the heart-rate, pulse, blood pressure and cardiac output in a more stable format. Hence,the cardiac output and the other form of pressure can be accompanied by means of determining the amount of blood being pumped out of the heart.

\section{E. Bandstop filters}

Depending upon the frequency range of the respiratory artifacts, they are considered as a low-frequency disturbance. Thus the high pass filters can be used thus the low frequency components can be get lost. The band-pass filters can filter out only the signal components at the respiratory-frequency. The normal respiration takes the minimum time of 8-30 breath per minute. A bandstop filter can remove the respiration at the frequency range of stopband between 1/7.5 and $1 / 2 \mathrm{~Hz}$. Due to the smaller capacity of the stop band.

\section{F. Adaptive filtering}

Let the measured blood pressure signal can be represented as $\mathrm{x}(\mathrm{t})$ and the correct blood pressure signal as $y(t)$ and a disturbance $v(t)$ caused by respiration.

$$
\mathrm{x}(\mathrm{t})=\mathrm{y}(\mathrm{t})+\mathrm{v}(\mathrm{t})
$$

Thus, the frequency of respiratory $\mathrm{f0}(\mathrm{Hz})$ can be established from the signal of respiratory and it is used to model the respiratory disturbances in the blood pressure.

And the approximation of the correct blood pressure $\mathrm{x}(\mathrm{t})$ is then

$$
\mathrm{x}(\mathrm{t})=\mathrm{s}(\mathrm{t})-\mathrm{v}(\mathrm{w}, \mathrm{t}) .
$$

Thus, $\mathrm{x}(\mathrm{t})$ represents the corrected blood pressure.

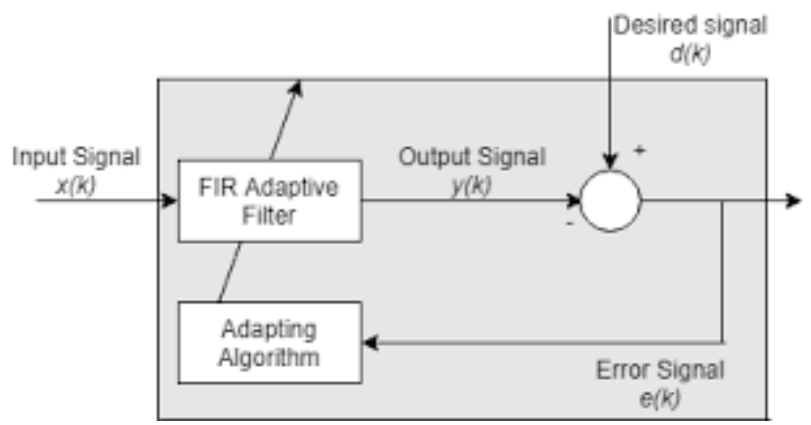

\section{Fig.7.adaptive filter}

\section{G. Wavelet method}

A wavelet transform method is a waveform which has limited duration with average value zero. Though the fourier analysis has a sine waves with smooth predictable waveforms with unlimited duration. So that the wavelets are irregular and assymetric. The fourier analysis is the concept of breaking up of sine waves with different range of frequencies at various conditions of signals. Like this manner in the wavelet analysis, the wavelet signal can be broken into scaled and translated signals. During the ventricular contraction there will be a sudden change in wavelet which is irregular with a smooth sinusoid.

\section{RESULT AND DISCUSSION}

Four different implementation has to be followed and these has to be implemented in MATLAB. The result of all kind of pressure can be obtained.

The variations in systolic and diastolic pressure at the respiratory frequency measured and will be compensated by means of this compensation technique. To determine the compensation of the signals, the spectra must be calculated. In which the spectra must be calculated before and after the rate of compensation which can be defined as,

Compensation $(\%)=100(1-(\varphi \operatorname{Comp}(\omega 0) / \varphi \operatorname{Org}(\omega 0)))$

where, $\varphi$ Org is the original spectra, $\varphi$ comp is the compensated and $\omega_{\omega 0}$ is the respiratory frequency.

TABLE 1:Compensation of heart beat rate

\begin{tabular}{|l|l|lll|}
\hline & \multirow{2}{*}{ Length } & \multicolumn{3}{|c|}{ Compensation(\%) } \\
& & SP & EDP & BDP \\
\hline Ventricular 1 & 10 & 81 & 75 & 86 \\
\hline Arterial 1 & 20 & 60 & -197 & - \\
\hline
\end{tabular}
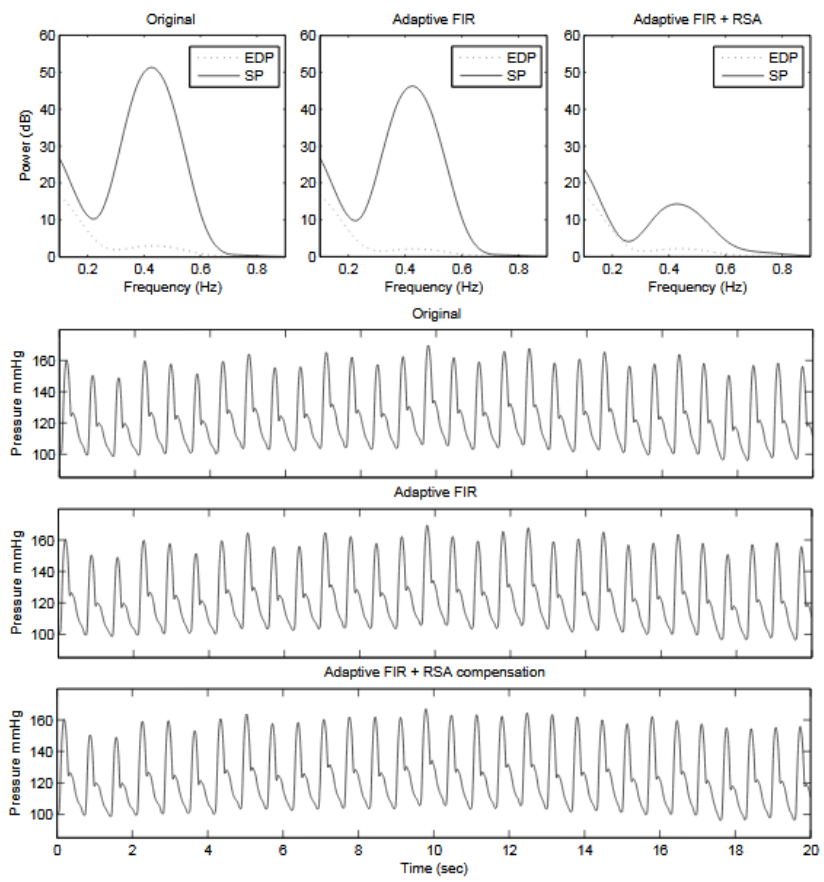

Fig:8 Resultant waveforms with removal of artifacts

Published By:

Blue Eyes Intelligence Engineering \& Sciences Publication

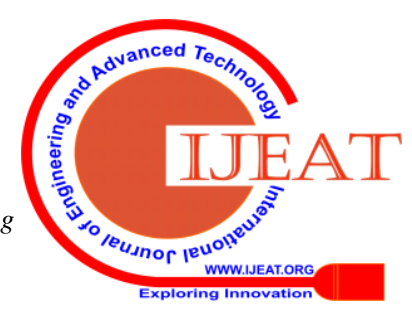




\section{CONCLUSION}

In this technology, four different types of methods have been implemented for the task of respiratory artifacts removal. In relaxation phase different respiratory variations systolic should be determined, by which the contraction phase of the heart in diastole can be obtained. The removal of respiratory artifacts can be accompanied by the simple filtering methods. Adaptive algorithms can be used to adjust the diastolic values and to filter out or to remove the actual intra-thoracic respiratory artifacts. If the frequency of respiratory varies, the algorithms might takes few time to adapt a new frequency of respiration in which correct result has been displayed. The wavelet compensation can demonishes all of the artifacts in the certain range of frequencies, thus the respiration and its deviations in the systolic pressure forms can produces a good and smooth waveforms, which can be resulted from all of the tested and generated signals.

\section{REFERENCES}

1. Reza Malekain and Johan Wannenburg.Body Sensor Network for Mobile Health Monitoring, a diagnosis and Anticipating System,2015.

2. Mary X.A, A.M Jegan \& Johnson. Performance on blood pressure and heart-rate measurement from PPG signal for biomedical applications,2017.

3. Z. Zhou, L.Xu, K.Chen and X.Zhang. A new method for locating the featured points in pulse wave using wavelet transform,2009.

4. M.Q.H. Meng Z. Shen, C.Hu. A pulse wave filter method based on wavelet transform soft-threshold and adaptive algorithm, 2010.

5. S.S. Ahrabi. Mathematical modeling of blood flow through an eccentric catheterized artery, 2014.

6. S.I. Zhen-Zhen. Application of Fourier transform and wavelet transform in signal de-noising, 2011.

7. L.Kong. MATLAB Wavelet Analysis Super Learning Handbook,2014.

8. J.R.C Jansen, S.A Hoeskel, J.A Blom and J.J Screuder. Correction in the respiratory artifact in pulmonary blood pressure signals of its ventilated patients, 1996.

9. K.H. Chon, M. Elstad, K.Toska, E.A. Raeder and R.J Cohen. Respiratory sinus arrhythmia which is opposite effects on systolic and mean arterial pressure in supine humans, 2001.

10. E.Ruokonen, I.Korhonen, J.Parkka and J.Takala. Method for estimation of pulmonary capillary pressure during intensive care, 1999.

11. N.K. Tabirkheli, S.T. Higano, E.Azrak and M.J. Kern. Influence of respiratory dynamics on ventricular pressures, 1999.

\section{AUTHORS PROFILE}

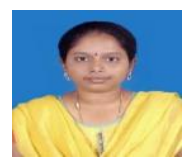

Shalini.S ME, is working as Assitant professor of ECE department in Sri Krishna College of Technology, Coimbatore. Her area of nterest is Embedded Systems and IOT.

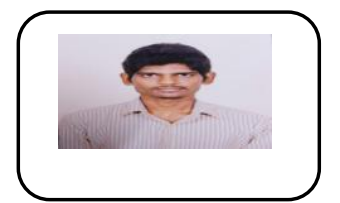

Mutamilan S, is studying an Electronics and communication Engineering in Sri Krishna College of Technology, Coimbatore. His area of interest is Embedded Systems.

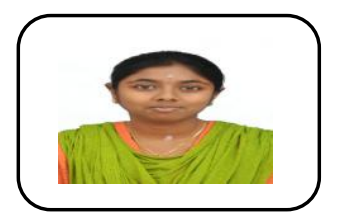

Monisha R, is studying an Electronics and communication Engineering in Sri Krishna College of Technology, Coimbatore. His area of interest is Embedded Systems.

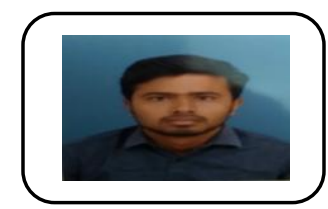

Nithish $\mathbf{M}$, is studying an Electronics and communication Engineering in Sri Krishna College of Technology, Coimbatore. His area of interest is Automation Electronics. 